\title{
Recurrent urticaria caused by specific cat serum albumin IgE cross-reacting with pork serum albumin
}

\author{
Key message \\ Question: What should be considered in children who com- \\ plain of pork allergies? \\ Finding: History of raising a cat, the onset of symptoms after \\ the ingestion of pork and specific IgE tests to pork, cat, milk, \\ and Alpha-gal are needed. \\ Meaning: Pork cat syndrome could be the cause of pork \\ allergies.
}

A 6-year-old boy presented with a 6-month history of recurrent urticaria after eating pork. He had allergic rhinitis to cat fur without drug allergy and a history of walnut anaphylaxis that had occurred 7 months previously. His parents-who owned a butcher shop—also had allergic rhinitis, and his older sister had bronchial asthma and allergic rhinitis. The family frequently ( $~ 5$ times/wk) ate chicken, pork, and beef that was grilled in a pan, with pork belly being their preference. In 2015, 2017, and 2018, the family kept a cat in the house for 1-3 months until the boy developed a cat allergy. Notably, he had exhibited no symptoms after eating pork before 2019. However, in 2019, he had experienced repeated urticaria and itching after eating pork, although these symptoms were not observed after he consumed chicken, beef, or milk.

On this occasion, 30 minutes after he had ingested pork, urticaria accompanied by an itching sensation developed in his hands and feet and later spread to his arms and legs. There was no coughing, gastrointestinal symptoms, or dyspnea. The symptoms resolved spontaneously 1 hour later; his physical examination and vital signs were unremarkable. There was no evidence of tick bite. Laboratory values at this visit and 7 months previously are presented in Table 1 . We did a prick-to-prick test with raw pork and grilled pork (Fig. 1) and an oral food challenge (with informed consent) to identify pork allergy. We started with 5 g of grilled pork belly; he showed no symptoms, but 20 minutes later, urticaria appeared on his trunk. We gradually increased the ingestion of pork to $27 \mathrm{~g}$ at 40 minutes. After 1 hour, he started to sneeze with conjunctival injection. He also complained of a febrile sensation, but his vital signs were normal. We stopped the test, and the symptoms resolved 1 hour later.

This study was approved by the Busan St. Mary's Hospital's Institutional Review Board (BSM2019-11).
Based on the history and clinical findings, which of the following is the most likely cause of this patient's urticaria?

(1) Pork-cat syndrome

(2) Alpha-gal syndrome

(3) Pseudoallergy

(4) Meat sensitivity by milk allergy

Table 1. Laboratory findings and reference ranges

\begin{tabular}{|c|c|c|c|}
\hline \multirow[b]{2}{*}{ Laboratory test } & \multicolumn{2}{|c|}{ Patient values } & \multirow{2}{*}{$\begin{array}{l}\text { - Reference } \\
\text { range }\end{array}$} \\
\hline & $\begin{array}{l}7 \text { Months before } \\
\text { the visit }\end{array}$ & $\begin{array}{l}\text { At the } \\
\text { visit }\end{array}$ & \\
\hline Eosinophil count $(/ \mu \mathrm{L})$ & 520 & 500 & $0-350$ \\
\hline Total lgE (U/mL) & 2,323 & 2,560 & $0-90$ \\
\hline $\begin{array}{l}\text { Dermatophagoides pteronyssinus } \\
\text { (kUA/L) }\end{array}$ & 44.90 & & $0-0.35$ \\
\hline Dermatophagoides farinae (kUA/L) & $>100$ & & $0-0.35$ \\
\hline Cat (kUA/L) & $>100$ & & $0-0.35$ \\
\hline $\operatorname{Dog}(k \cup A / L)$ & 31.60 & & $0-0.35$ \\
\hline Walnut (kUA/L) & 10.50 & & $0-0.35$ \\
\hline Milk (kUA/L) & & 0.54 & $0-0.35$ \\
\hline Beef (kUA/L) & & 0.82 & $0-0.35$ \\
\hline Pork (kUA/L) & & 8.68 & $0-0.35$ \\
\hline Chicken (kUA/L) & & 0.21 & $0-0.35$ \\
\hline Casein (kUA/L) & & 0.24 & $0-0.35$ \\
\hline Fel d $2^{\text {a) }}(\mathrm{kUA} / \mathrm{L})$ & & 61.40 & $0-0.35$ \\
\hline Bos d $6^{b)}(k U A / L)$ & & 1.00 & $0-0.35$ \\
\hline Alpha- gal ${ }^{\mathrm{c})}(\mathrm{kUA} / \mathrm{L})$ & & 0.03 & $0-0.35$ \\
\hline
\end{tabular}

IgE, immunoglobulin E.

a) Specific IgE to cat serum albumin. ${ }^{b}$ Specific IgE to cow serum albumin.

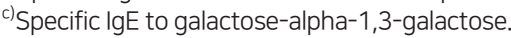

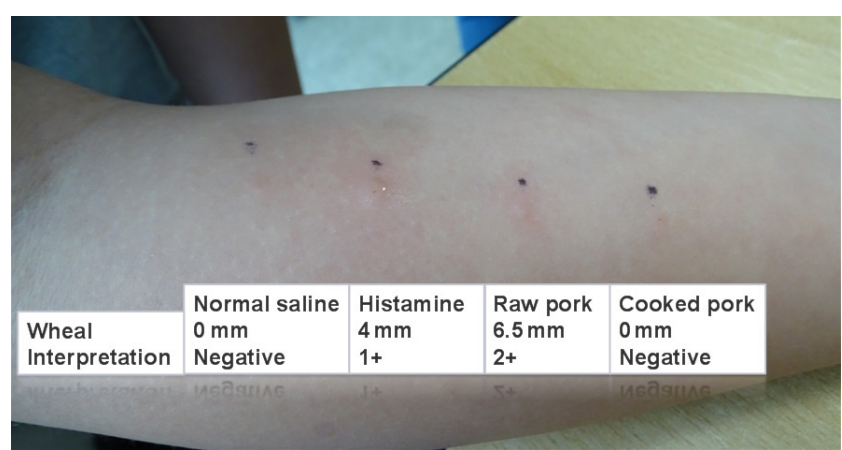

Fig. 1. Results of the prick-to-prick test with raw versus cooked pork. A positive result was observed for raw pork versus a negative result for cooked pork, suggesting that the allergenic component was thermolabile. 


\section{What is pork-cat syndrome?}

Pork-cat syndrome (PCS) is an uncommon allergic reaction to pork serum albumin (SA; allergen, Sus s 1 ) and occurs secondary to sensitization to cat SA (allergen, Fel d 2). It is a meat allergy caused by cross-reactivity to allergens from different mammal species. The route of Fel d 2 exposure is mainly the airway. Currently, Fel d 2 sensitization is considered to always precede Sus s 1 sensitization. ${ }^{1)}$ Because SA is thermolabile, well-cooked meat is less allergenic. ${ }^{2)}$ There have been a few reports of PCS. The first PCS case was described by Drouet and Sabbah in 1994.") Posthumus et al. ${ }^{4)}$ described eight cases of PCS in the United States. Symptoms vary from anaphylaxis to urticaria. Previously reported cases have mostly involved adults or adolescents. To diagnose PCS, detailed history of reaction to pork within 1 hour of ingestion and results of the skin prick test; specific immunoglobulin E (sIgE) tests for pork, cats, beef, Sus s 1, and Fel d 2; and food challenge test are needed. ${ }^{2)}$ Because the sIgE to Fel $\mathrm{d}$ 2 could decrease over time, avoiding exposure to pork and cats is currently the treatment of choice..$^{5)}$ In this case, the patient presented a typical history of food allergy to pork, positive food challenge test results, positive prick-to-prick test results for raw pork, and sIgE to Fel $\mathrm{d} 2$ and pork IgE. Hence, the diagnosis was PCS. Parents were advised not to give him pork and to avoid cats. His symptoms resolved thereafter.

\section{How to distinguish Pork-cat syndrome from other meat allergies?}

PCS is an IgE-mediated hypersensitivity reaction involving Fel d 2 and Sus s 1. ${ }^{6}$ In general, symptoms begin within 1 hour after eating pork. By contrast, in alpha-gal syndrome, caused by tick bite, symptoms start after 3-6 hours after exposure due to delayed anaphylaxis reaction. It is because of the presence of sIgE for galactose-alpha-1,3-galactose (alpha-gal) in red meat. ${ }^{7)}$ If a patient has an allergy to antibiotics such as penicillin or streptomycin, the antibiotics present in meat can mimic meat allergy, although the cause is not meat allergy but allergy to antibiotics. ${ }^{8)}$ Patients with milk allergy occasionally develop sensitivity to meat. In those cases, patients could have sIgE for milk, casein, and Bos $\mathrm{d} 6$ (cow SA). ${ }^{2)}$ In our case, the patient tolerated milk well, and had just low level of sIgE for milk and negative sIgE for casein. Hence, alpha-gal syndrome, meat sensitivity due to milk allergy, and pseudoallergy were excluded.

\section{Why is there a cross-reaction between cat serum and pork serum?}

All mammalian SAs have a conserved protein structure and share similar amino acid (AA) sequences, resulting in homology.9) Comparisons of AA sequences to identify cross-reactivity among allergens are generally used. ${ }^{10)}$ In our inspection, we listed com. mon mammalian SAs and their AA sequence from NCBI (https:// www.ncbi.nlm.nih.gov/): Can f 3 ( $\operatorname{dog}$ SA), Fel d 2, Bos d 6 (cow SA), Gal d 5 (chicken SA), and Sus s 1. We then compared them using a sliding window of $80 \mathrm{AA}$ segment alignments by FASTA in Allergenic Protein Sequence Searches (http://www.allergen online.com/databasefasta.shtml), which are generally used to predict allergen cross-reactivity. Fel d 2 shares $87.00 \%$ of its AA sequence with Can $\mathrm{f} 3,79.40 \%$ with Sus s $1,78.30 \%$ with Bos $\mathrm{d} 6$, and $46.50 \%$ with Gal d 5. This might help explain why cat SA sensitization can cause cross-reaction to pork rather than to chicken.

\section{Answer: (1) Pork-cat syndrome}

\section{Conflicts of interest}

No potential conflict of interest relevant to this article was reported.

\section{Cheon Kim, MD, Sung Won Kim, MD, Yoon Ha Hwang, MD \\ Department of Pediatrics, Busan St. Mary's Hospital, Busan, Korea}

Corresponding author: Yoon Ha Hwang, MD

Department of Pediatrics, Busan St. Mary's Hospital 25-14, Yongho-ro, 232beon-gil, Nam-gu, Busan 48575, Korea

凶E-mail: hyh190@naver.com,

https://orcid.org/0000-0002-6508-8168

\section{References}

1. Hilger C, Kohnen M, Grigioni F, Lehners C, Hentges F. Allergic crossreactions between cat and pig serum albumin. Study at the protein and DNA levels. Allergy 1997;52:179-87.

2. Matricardi PM. EAACI molecular allergology user's guide. Pediatr Allergy Immunol 2016;27 Suppl 23:117-8

3. Drouet M, Sabbah A. The pork/cat syndrome or crossed reactivity between cat epithelia and pork meat. Monogr Allergy 1996;32:164-73.

4. Posthumus J, James HR, Lane CJ, Matos LA, Platts-Mills TA, Commins SP. Initial description of pork-cat syndrome in the United States. J Allergy Clin Immunol 2013;131:923-5.

5. Savi E, Rossi A, Incorvaia C. Cat-pork syndrome: a case report with a three years follow-up. Eur Ann Allergy Clin Immunol 2006;38:366-8.

6. Posthumus J, Workman LJ, James HR, Pochan SL, Lane CJ, McBride $\mathrm{DC}$, et al. In the pork-cat syndrome, allergic reactions to pork reflect IgE antibodies to cat albumin that cross-reacts with other albumins. J Allergy Clin Immunol 2012;129:AB177.

7. Commins SP, Satinover SM, Hosen J, Mozena J, Borish L, Lewis BD, et al. Delayed anaphylaxis, angioedema, or urticaria after consumption of red meat in patients with IgE antibodies specific for galactose-alpha-1,3galactose. J Allergy Clin Immunol 2009;123:426-33.

8. Raison-Peyron N, Messaad D, Bousquet Jean, Demoly P. Anaphylaxis to beef in penicillin-allergic patient. Allergy 2001;56:796-7.

9. Chruszcz M, Mikolajczak K, Mank N, Majorek KA, Porebski PJ, Minor W. Serum albumins-unusual allergens. Biochim Biophys Acta 2013; 1830:5375-81.

10. Song P, Herman R, Kumpatla S. 1:1 FASTA update: using the power of E-values in FASTA to detect potential allergen cross-reactivity. Toxicol Rep 2015;2:1145-8. 
How to cite this article: Kim C, Kim SW, Hwang YH. Recurrent urticaria caused by specific cat serum albumin IgE cross-reacting with pork serum albumin.Clin Exp Pediatr 2020; 63:451-3. https://doi.org/10.3345/cep.2019.01725 